\title{
humanidades
}

Revista humanidades

Enero-junio, 2016•Volumen 6, número 1 • ISSN 2215-3934•pp. 1-43

\section{¿Qué es Tecnología? Una aproximación desde la Filosofía: Disertación en dos movimientos}

DOI: http://dx.doi.org/10.15517/h.v6i1.25113

\section{Julio Ernesto Rubio Barrios}

Doctor en Filosofía y Ciencias de la Educación. Director de la Escuela de Ciencias Sociales y Humanidades en la Ciudad de México del Tecnológico de Monterrey, México. Correo electrónico: jerb@itesm.mx

\section{Rodrigo Esparza Parga}

Doctor en Estudios Humanísticos con Especialidad en Ciencia y Cultura. Profesor Investigador de Cátedra del Tecnológico de Monterrey, Campus Ciudad de México y Campus Santa Fe. Correo electrónico: fil.rodrigo.esparza.parga@itesm.mx

Todos los derechos reservados. Universidad de Costa Rica. Esta revista se encuentra licenciada con Creative Commons Reconocimiento-NoComercial-SinObraDerivada 3.0 Costa Rica.

Correo electrónico: humanidades@ucr.ac.cr / Sitio web: http://revistas.ucr.ac.cr/index.php/humanidades 


\title{
¿Qué es Tecnología? Una aproximación desde la Filosofía: Disertación en dos movimientos
}

\section{Resumen}

Iniciamos este tránsito del pensamiento preguntando por el qué es la tecnología. Y este habrá de ser nuestro constante preguntar a lo largo de las siguientes líneas; todo ello, con miras a elucidar una sucinta aproximación al ser esencial, al ser que insufla el carácter propio de aquello que nominamos Tecnología. Afirmamos que en tanto que humanos, nos resulta imposible escindir de dicha condición eso que llamamos Tecnología. Esta ha de ser la tesis fundamental que a través de las siguientes líneas se pretende defender Identificamos dos elementos esenciales que subyacen a toda tecnología, y estos son el transformar y el producir y tales categorías se habrán de desarrollar con miras a clarificar el qué de esa dimensión llamada Tecnología. Hacer un esfuerzo genuino por ofrecer una respuesta integral y coherente, mas no acabada, a la no sencilla pregunta de ¿qué es la tecnología? representa en la actualidad un imperativo, un hacer que no se puede postergar más, debido a las repercusiones que el ejercicio tecnológico ha tenido, tiene, y habrá de tener, sobre la especie y el lugar que la especie ocupa en la existencia, es decir, el mundo.
Palabras clave: Filosofía de la tecnología, tecnología, óntica tecnológica, transformar, producir.

\section{What is Technology? An Approach from Philosophy: Dissertation in two Movements}

\begin{abstract}
We begin by asking what technology is and it is a sustained question throughout the text. The purpose is to search for a brief approximation to the essence of being which in turn refers to technology. We ascertain that humans cannot exist without the condition of technology. This is the fundamental thesis which identifies two essential elements that underlie technology: transformation and production. These categories aim to explain the meaning of the so-called technological dimension. To make a genuine effort to offer a holistic and coherent answer to the simple question of what is technology represents an imperative, a to do that can no longer wait due to the repercussions that technology has had, has and will have on the human species and the place they occupy in the existence, that is, in the world.
\end{abstract}

Keywords: Philosophy of Technology, technology, technological ontic, transforming, producing. 
Iniciamos este tránsito del pensamiento preguntando por el qué es la tecnología. Y este habrá de ser nuestro constante preguntar a lo largo de las siguientes líneas; todo ello, con miras a elucidar una sucinta aproximación al ser esencial1, al ser que insufla el carácter propio de aquello que nominamos tecnología.

Afirmamos que en tanto que humanos, nos resulta imposible escindir de dicha condición eso que llamamos tecnología. Esto ha de ser la tesis fundamental que a través de las siguientes líneas se pretende defender a partir de argumentos de diversa índole, pero todos ellos, se puntualiza, insertos en una perspectiva filosófica, que por ciertos momentos matizará hacia lo histórico y por otros con lo sociológico, empero, siempre, siguiendo, creciendo en torno a un eje de naturaleza filosófica.

Toda vez que el ser humano como especie hace acto de presencia en la existencia, se relaciona de manera inexorable con aquello que no es él, es decir con el exterior, con el medio, entendiendo por este, el mundo de los otros hombres, las realidades materiales por estos creadas y el mundo propiamente natural.

Históricamente, como especie, hemos hecho, hemos traducido y adaptado; hemos transformado y producido. Adaptado el afuera, que ya se mencionó; el que no es yo, nosotros, es decir, el medio, id est, los otros hombres, las realidades materiales por estos creadas y el mundo propiamente natural:

A aquellos lugares para llegar a los cuales el hombre se pasaba semanas o meses viajando se llega ahora en avión en una noche. Aquello de lo que el hombre no se enteraba más que pasados unos 
años, o no se enteraba nunca, lo sabe ahora por la radio, todas las horas, en un abrir y cerrar de ojos (Heidegger, 1994, p. 143).

La cita anterior es testimonio fehaciente de lo que uno de los prominentes filósofos occidentales del siglo XX pronunció en torno a lo que líneas más arriba señalamos en el sentido de que la transformación de lo que "natural" otrora fue, por el humano ha sido trasegado, mutado y reconfigurado de modo tal, que es, en primera instancia, manifestación, objeto de fin y uso, nos referimos al medio, o sea, a los otros hombres, al mundo material por estos creado y al mundo propiamente natural.

Y señalamos manifestación pues, todo el constructo humano, es la proyección de su interior que encuentra eco en el exterior, en el cual toma forma, corpus y se incorpora al mundo humano y se funde con el natural, y en los cuales, y con tal arribo, se amplían las posibilidades, desde el interior del hombre, hacia el exterior de los hombres. Es para decirlo de otra manera, que "el contenido del espíritu se descubre solo en su manifestación; la forma ideal es reconocida solo en y por la totalidad de los signos sensibles de los cuales se sirve para expresarse" (Cassirer, 1971, p. 28). Y dichos signos sensibles dan corpus al ámbito de lo simbólico, de la representación, para finalizar en la materialización de aquello otrora simbolizado, representado.

El tiempo de la espera se ha reducido y la cercanía lo está más que anteriormente. El mundo, definitivamente, no es el mismo y no lo será en lo sucesivo, y no lo será en la medida no solo en que de por sí es cambiante, sino que hay un factor que se suma a su ya cambiante devenir, y tal factor resulta ser una suerte de catalizador de tal devenir, y aquél es el ser humano. 
Transformando y produciendo, según sus propias directrices, modificando lo que de suyo es cambiante, el mundo; añade a este rápidamente un nuevo modelado, reconfigurándolo según su ser-necesario; según su ser-saber; según su ser-acción y finalmente; según su ser-sensible, es decir, según cierta inexorabilidad, cierto tipo de saber, cierto tipo de acción y finalmente, cierta sensibilidad. Nos referimos al ser-necesario, como a la imposibilidad de que el humano no genere tecnología; al ser-saber, en la medida en que la tecnología es un corpus de conocimiento con sus características propias; al ser-acción en tanto que la tecnología es un facio hominis; y finalmente, al ser sensible en la medida en que la tecnología posee una dimensión estética.

Hemos moldeado, modificado a placer el mundo, en el sentido extenso de la palabra. No solo para protegernos o salvaguardarnos, sino que hemos impreso en esas transformaciones el carácter de lo eficiente y de lo ineficiente, pero también, el de lo placentero, del gusto, de lo agradable y de lo desagradable. El conseguir tal adaptar, tal transformar y que esto último resulte no solamente eficiente, sino también placentero, sea a la vista, al gusto o a cualquiera de nuestros sentidos, requiere de un saber, de un conocer, es decir de una relación, de una interacción entre el sujeto y el objeto. Relación desde la cual el sujeto, sustrae, aprehende las cualidades del objeto.

Con ello, se aprende, se conoce sobre los objetos y el mundo, y en una segunda instancia, se entiende el quid de cada uno de aquéllos y del mundo, finalmente. Sapiente de lo anterior, el humano, da un giro a tal saber para ser este utilizado a fin de generarse las condiciones propiamente humanas, tales que, le permitan vivir humanamente. 
En un segundo momento, se ha iniciado un transformar, un producir, con miras a ya no solo a través de tales haceres el proveer las condiciones fundamentales de la vivencia, de la existencia orgánica, sino a ser propiamente ser humano, es decir, a proyectar, a materializar todo aquello que a través de la imaginación y la estructura simbólica sea posible llevar al cabo.

Es lo anterior posible gracias a la tecnología, por lo que afirmamos, que no podemos no elegir a la tecnología como forma de vida, y en tal sentido es una condición necesaria para la especie humana -trataremos dicho carácter necesario en una posterior publicación. Pero en un sentido estricto, ¿qué es la tecnología?, ¿qué es lo tecnológico?, ¿cuáles son las características presentes en aquello que calificamos como tecnológico?

Procuremos pues, elucidar algunas respuestas a tales cuestionamientos. Haremos lo anterior, a partir del desmenuzar la noción de tecnología en diversas dimensiones, tantas como nuestro análisis filosófico nos lo permita.

Notamos en primera instancia, cuatro dimensiones fundamentales sobre la tecnología, a saber, una dimensión óntica, una epistémica, otra ética ${ }^{2}$ y una más de carácter estético.

Dícese ente de lo que es, o para decirlo con Heidegger, "ente es todo aquello de lo que hablamos, lo que mentamos, aquello con respecto a lo cual nos comportamos de esta o aquella manera; ente es también lo que nosotros mismos somos, y el modo como lo somos" (Heidegger, 2002, p. 30) Y vaya que la tecnología es algo de lo que ahora hablamos, mentamos

Óntica tecnológica o de la pregunta por el ser de la tecnología y nos comportamos con respecto a ella, nos insta a cierta acción-reacción, 
es, existe; empero todo lo que existe es ente, sea abstracto o concreto, y aún así cada ente es uno y particular, aunque compartiendo la categoría de lo ente, entonces ¿de qué manera es posible diferenciar toda la diversidad de entes y no perdernos en una multiplicidad indiferenciada?

Así pues, se considerará a la tecnología en lo sucesivo, como un ente, es decir, un "algo" sobre el cual es posible identificar una necesidad de reflexión pronta y profunda -asunto a tratarse en una posterior publicación- debido a que se encuentra de manera tan presente y actual, que el primer contacto a través de los sentidos, es decir hacia el exterior, alguno de ellos, u otros más en conjunto, tienen como percepción primera un objeto concreto de naturaleza tecnológica.

Tales objetos, son tan variados y de tan diversa índole que primero nos cansaríamos antes que poder mentarlos a todos. Un hospital, una casa, un respirador, una transfusión, los auxilios en el proceso mismo del parto y una cantidad de más ejemplos, podrían poner de manifiesto aquello que hemos señalado en las líneas anteriores. Por ello es que se considera pertinente la pregunta sobre el ¿qué es la tecnología?

Si otrora se estuvo en Occidente sumergido en un "mundo religioso" y los temas de fondo filosóficos tuvieron que ver con tal temática, por qué no, tendría que ser objeto de intensa reflexión la cuestión tecnológica, aún más debido a que la misma no se nos presenta de fondo siquiera como explicación del mundo en sus dimensiones todas, sino más bien, como una pragmática ineludible. Errónea determinación sería el que se aleje el asunto como objeto de discusión si es que a través de la tecnología es que se han gestado nuevas formas de nacer, de "entrar" en la vida, en el asunto del 
ser-siendo-en-el-mundo-tangible, y con ello, con la consecuente diversidad de posibilidades de lo concreto.

Mas menester es el considerar que a la vez que las extensiones de "el nacimiento, lo nacido" han encontrado nuevas formas de proyección, de hacer acto de presencia; también se han multiplicado las formas de morir, "la muerte, lo muerto".

Se han diversificado las posibilidades de hacerse presente; vida y muerte se hacen presentes de nuevas formas a través de la tecnología. V. gr., la inseminación "artificial” está presente en el sentido de las "nuevas formas posibles de nacer", por el contrario, la ruptura de un arnés, el derrumbe de un edificio, o el fallido despegue de un cohete, lo están como las "nuevas formas posibles de morir".

Pero el preguntar por la tecnología, y la posible respuesta, trasciende lo señalado anteriormente, y para responder a nuestra pregunta, habrá que considerarla como un algo que posee características que la delimitan y con ello, por consecuencia, nos es posible diferenciar y definir del resto de los entes.

Su carácter óntico pues, se cuestionará a partir del qué es la tecnología y en ese afán de consecución es que trataremos de esbozar una óntica tecnológica que nos clarifique el quid de esta, sus alcances, posibilidades y limitantes, o hasta qué punto, el humano a través de esta, encuentra su verdadera potencialidad y con ello, su extensión vivencial plena.

Antes de procurar definir, tendremos que hacer un esfuerzo reflexivo en pos de ir tras aquello que por esencia tiene este ente nominado tecnología, 
además de esas características que le otorgan la diferencia específica, lo que nos permitirá establecer un punto de partida en la construcción de una noción sobre lo que la tecnología es, y a lo que a esta atañe.

Los conceptos parece que los tenemos claros hasta que los cuestionamos de manera rigurosa. Lo que tenemos claro, es en sí una sucinta aproximación al concepto, alguna vaga idea de lo que este conceptualiza.

Así, si es que nos cuestionamos ¿qué es la tecnología?, no sabemos lo que esta es, empero, nuestro pensamiento se orienta hacia una serie de nociones que tienen qué ver con esta y se establecen ciertas relaciones entre las primeras, que, en su conjunto, nos permiten aproximarnos a una respuesta válida para la pregunta ¿qué es la tecnología?

Iniciemos pues, con la revisión de algunas nociones de tecnología, tal que su profundo análisis, nos permita llegar a una idea clara de lo que habremos de entender por ella.

En su libro Tecnología autónoma Langdon Winner apunta que:

[La tecnología] en la actualidad, es ampliamente usada en el lenguaje académico y en el común para referirse a un conjunto increíblemente variado de fenómenos; herramientas, instrumentos, máquinas, organizaciones, métodos, técnicas, sistemas y la totalidad de todas estas cosas y otras similares en nuestra experiencia (Winner, 1979, p. 19). 
Si bien es cierto que no es el mismo Winner quien nos expone su propia noción de tecnología, sino que solo nos dice que en la actualidad es ampliamente usada en el lenguaje académico y en el común, podemos observar que esta aproximación a la noción de tecnología resulta sumamente común, es decir, es a lo que se suele apelar como respuesta cuando se cuestiona sobre el qué es la tecnología.

Tiene sentido dicha respuesta en la medida en que sí nos señala cuáles serían los derivados de la tecnología, o en otras palabras, su frutos, o evidencias, mas no nos dan cuenta de lo que es en sí la tecnología. Mas tal definición pierde su sentido cuando el concepto se lo nutre de otros de modo que se torna sumamente amplio para finalmente, pretender afirmar que todo es tecnología y por tanto lo definido pasa a ser todo y nada, y con ello, a la indefinición.

No cuestionamos por alguna particularidad de la tecnología, sino lo hacemos en su sentido amplio, lo que en tanto que ente le compete, pero siempre procurando la delimitación adecuada de modo que no se convierta en un vertedero de nociones que terminen por decirnos nada sobre la tecnología. Buscamos el fundamento de esta y nos cuestionamos a la vez, cuál habrá de ser la tendencia de dicho fundamento.

Si en un primer paso apelamos a la etimología del concepto tecnología, proviene este, de las nociones griegas de $\tau \dot{\varepsilon} \chi v \eta^{3}$ “que no significa ni arte ni técnica sino «saber», disposición sapiente de la libre planificación y organización y el dominio sobre lo organizado" (Heidegger, 1997, p. 25) y $\lambda o \gamma o \varsigma^{4}$ (razón o tratado) de lo que podríamos interpretar que la tecnología sería algo así como el discurso en relación al cómo hacer, y el hacer en dos sentidos, uno artístico y otro artesanal o técnico, pero en cuyas nociones 
finalmente, se encuentra la idea de transformación, de modificación del medio, del entorno, a través del seguimiento de cierta instrucción previa o planeación y sobre la organización de esto último.

Pero por el momento, dejemos de lado las dos ideas de transformación a las cuales nos remite la noción de tecnología y volquémonos sobre lo que su etimología nos ha de implicar.

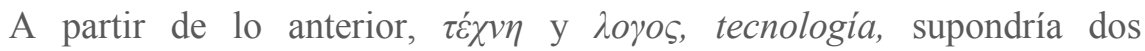

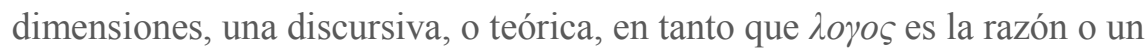
"decir algo sobre"; lo que estaría suministrando cierto "conocimiento sobre los objetos de acción (...)" (Bunge, 2004, p. 64) y en tal sentido desvelando el objeto mismo o al menos su posibilidad y con ello ampliando extendiendo el plano del ser, pues del no ser, del no existir se desentiende para ubicarse dentro de la existencia y con ello, nutrir la predicación del Ser; y la otra dimensión de orden práctico o de acción estaría presente, en la medida en que $\tau \dot{\chi} \chi v \eta$ es “creación y construcción, en tanto producción a partir de un saber" (Heidegger, 1997, p. 25) o sea que, es aquel hacer que (se) "produce a partir de un decir cómo" o bien, "porque se refiera a la acción misma" (Bunge, 2004, p. 64). Es decir, a esa acción que a lo largo de su desarrollo, siguiendo la guía de la tekne, estaría conduciendo a su ejecutor a transitar desde de la "ahora idea posible", a la materialización de la misma en el ámbito del hecho a partir de la transformación y través de la producción, de modo que (...) lo traído-ahídelante de un modo artesanal ${ }^{5}$ [tecnológicamente] y artístico, por ejemplo la copa de plata, no tiene la eclosión del traer-ahí-delante en él mismo sino en otro ( $\varepsilon \vee \alpha \lambda \lambda \omega)^{6}$, en el artesano [el tecnólogo] y el artista” (Heidegger, 2007, p. 12). 
Es pues, que quien conduce tal despliegue de acciones, de secuencias, es el humano, hecho que ha efectuado desde que se considera como tal, por lo que podemos señalar que de lo anterior se desprende un fundamental antropológico, un primordial óntico del mismo.

El ser humano por esencia, transforma a través de la producción, transforma el entorno y se transforma a sí mismo. Y es a partir de tal transformar y producir, que plasma, que proyecta desde el ámbito de lo interno lo concerniente a sus necesidades de ser viviente, de ser trascendente.

"Humankind, since its inception, used tools and materials both to ensure its survival as well as to express freedom/self-realization. The essence of Homo faber is to control and manipulate nature to serve human ends (...)”. [La humanidad, desde su comienzo, ha utilizado tanto herramientas y materiales a fin de asegurar su supervivencia, así como para expresar su libertad y auto-realización. La esencia del Homo faber es el controlar y manipular a la naturaleza a fin de servir esta, a los fines humanos (...)]" (Lee, 2009, p. 13-4).

Proyecta, además, en ese despliegue interno que a través de la producción se transforma en diversas manifestaciones de carácter creativo que complementan su relación con el medio y con los otros, aquello que denominamos arte. “Así, nos vemos llevados a la inevitable conclusión (...) de que todo lo que procede del hombre [la tecnología misma] no es sino la propia naturaleza [esencia] humana que se autodisemina y que de este modo alcanza su verdadera conciencia (...)” (Kapp, 2001, p. 129)

De manera que lo más propio del ser humano sería, según Kapp a partir de la cita señalada en López et al. (2001), aquello que a través de la tecnología 
cobra materia, forma, llevando de aquí a allá ese modo de ser, que a través de su manifestación permitiría cobrar consciencia al humano, y en esta materialidad informada y manifiesta, reencontraría a cada instante la unidad material y espiritual de su propio ser.

Es en tal sentido, que a través de la tecnología se proyecta hacia el exterior del hombre aquello que en un primer momento solo concibe en el interior, y que una vez materializado, es objeto de conocimiento ya no solo por su generador, sino por "los otros" y es en tal sentido en que la interioridad queda expuesta, mostrando así, las diversas facetas que componen la consciencia del hombre.

Pero tomemos con cierta ligereza nuestro aserto, pues más delante expondremos los argumentos que permitirán conferir solidez a lo que por ahora solo planteamos como un esbozo.

Por otra parte, resulta que la transformación, a la que nos referimos líneas arriba, es realizada en base a cierta instrucción, apoyada en cierto saber sobre el objeto en potencia que orienta la acción. Acción que, a su vez, enriquece a través de su desarrollo al logos mismo sobre el objeto y en una suerte de espiral, logos nutre de nueva cuenta a la tekne y así sucesivamente.

Una noción común respecto a la tecnología, es la de medio, la de "un entramado humano de utensilios -herramientas, máquinas, instrumentos, materiales, ciencias y personal- que hacen posible y sirven a la construcción de unos fines por parte del hombre" (Hood, 2004, p. 480). 
Fines que no estarían en la tecnología misma, según la noción señalada por Hood, quien, a su vez, expone tal idea a partir de las explicaciones aristotélicas, que serían las que realmente darían cuenta de la manera tradicional de concebir a la técnica.

Sin embargo, nuestra tarea no se dirige hacia la búsqueda por el sentido y ser de la técnica, sino el de la tecnología, aunque para tal menester resulta necesario mencionar que la distinción semántica entre la una y la otra, representa un problema complejo y que parte, en gran medida, se deriva de la tradición en la cual se encuentre uno arraigado.

“The term 'technology' is not well defined, in English even less precisely than in German. Commonly, 'technology' means the practice and the results of engineering as well as the scientific research on engineering”. [El término "tecnología” no está bien definido, y en inglés es aún menos preciso que en alemán. Comúnmente "tecnología" significa la práctica y los resultados de la ingeniería, así como, de la investigación científica en ingeniería] (Rophol, G. 1997, p. 67).

Es así, como en la tradición alemana, y en palabras del mismo Rophol: “... we denote a knowledge as 'technical', when it applies to engineering practice, and as 'technological', when it applies to engineering science”. [Nosotros usamos la palabra "técnicas" para denotar el campo del trabajo ingenieril y sus productos] (Ropohl, 1997, p. 65). Por otro lado, el término "tecnología” será restringido a la ciencia de las técnicas, justo como Johann Beckmann (1777) hubo introducido el concepto" (Rophol, 1997, p. 67). 
En castellano el uso más común para referirse a lo que es objeto de nuestra investigación, resulta serlo el vocablo "tecnología", empero, Ortega y Gasset usó (1933) la palabra "técnica”, para referirse a lo que nos, nombramos como "tecnología", y ello, resulta claro comprenderlo del porqué lo hizo, si consideramos que la herencia intelectual por él recibida, fue en buena medida de naturaleza germana.

Así, como lo comenta Armando Chiappe (2009, p.52) en la tradición alemana se habla de una y otra indistintamente, utilizando para ello el vocablo Technik, mientras que, en la tradición anglo-sajona, suele hacerse la distinción entre Technic (o Technique) y Technology.

Por otra parte, y a fin de señalar algunas diferencias fundamentales que sí distinguimos efectivamente entre un concepto y otro, es que la técnica comporta "haceres" distintos a los que se implican en la tecnología, como, por ejemplo, la ejecución de una pieza musical al piano, que ha de requerir técnica por parte del ejecutor, no de tecnología, pero para hacer el piano sí que requerimos, en efecto, de tecnología, y el mismo piano, ha de ser una derivación tecnológica.

Otro distingo fundamental que identificamos, es que la técnica en su corpus cognitivo no desvela aspecto alguno del mundo, el corpus cognitivo de la tecnología, sí, pero esto habremos de abordarlo en otro artículo.

La técnica es un mero know-how, es "un conjunto de habilidades y conocimientos que sirven para resolver problemas prácticos” (Quintanilla, 2001, p. 56) Aunque el problema práctico sea, por ejemplo, interpretar una pieza musical con algún instrumento. 
Por último, y debido a que nuestra investigación es sobre la Tecnología, y no sobre la técnica, hemos de indicar que la técnica es un hacer humano y que como señala Chiappe (2009, p. 52) "lo normal es que con el vocablo técnica se haga referencia al ámbito procedimental, mientras que con el de tecnología, por su componente logos (reflexión), se pone en conexión praxis y conocimiento (el marco intelectual)".

Mas retomando el parafraseo aristotélico que expone Hood, señala que la técnica no es, pues, una actividad que satisfaga en sí misma la naturaleza humana; más bien, es algo que el hombre hace solo con vistas a arreglárselas para conseguir una cosa. No es un fin en sí misma sino simplemente un medio para otro fin ulterior. En otros términos, la técnica es extrínseca a la naturaleza humana (Hood, 2004, p. 480).

Tales aseveraciones son sumamente cuestionables, pues un característico de la esencia de algo, es que esta acompaña al objeto del cual es esencia a lo largo de su existencia, y la realización de dicha esencia, constituye la función o fin más propio de lo que por esencia es.

Si ha sido que el humano desde lo más remoto en el tiempo se identifica a sí mismo transformando y produciendo, ¿no es pues de suponer que eso es un fundamental, un principio constitutivo de lo esencialmente humano? No es posible para el ser humano sujetarse a la pasividad, tiene por naturaleza un impulso dinámico que se manifiesta a través del transformar y producir.

Por otra parte, la actividad tecnológica ha suplantado el ámbito del medio para instaurarse ella misma como un fin, al grado que ha conseguido el ser humano, inclusive, mayor satisfacción a partir de esta que la que la misma 
naturaleza le ha podido otorgar. Finalmente, aquello que constituye la esencia de algo, no puede ser el medio del algo, sino su fin mismo.

Por consiguiente, podemos afirmar que la tecnología no es un medio, sino un fin en sí misma. No cuestionamos por alguna particularidad de la tecnología, sino lo hacemos en su sentido más amplio, lo que en tanto que ente le compete. Buscamos el fundamento de esta y nos cuestionamos a la vez, cuál habrá de ser la tendencia de dicho fundamento, yendo así, tras la claridad que nos permita comprender lo que la tecnología es, podemos aproximarnos a través de lo que consideramos que no es.

Analizando la postura tradicional o aristotélica, Hood nos señala que "la técnica es un dispositivo humano de utensilios con vistas a la consecución de unas metas humanas, metas que son extrínsecas a ese dispositivo y están determinadas por el orden inteligible del cosmos que, a su vez, se refleja en la estructura estable de la sociedad" (Hood, 2004, p. 485), ante lo que no podemos sino alegar que, primeramente, la tecnología no es un "dispositivo de utensilios", aunque sí comprende a estos, empero no se reduce a ellos, comprende dimensiones allende a estos, por ejemplo, cuando hablamos de procesos e ideas mismas.

En nuestra actualidad los "utensilios" o demás objetos, producto de la tecnología, son en muchos casos fin en sí mismos y no meros "salvoconductos" con miras a la consecución de determinadas metas. Piénsese a modo de ejemplo, en el Proyecto Manhattan, por citar solo un caso. 
Por otra parte, tales metas señaladas, no siempre resultan ser extrínsecas al “dispositivo de utensilios", pues estos son utilizados en la producción de "otros utensilios", y finalmente, el orden de dichas metas humanas son tales, que se ven profundamente trastocadas por la tecnología, al grado tal, que históricamente visto está, que han provocado el rompimiento de la señalada "estructura estable de la sociedad".

Es de notar que la actualidad en la que vivimos, la tecnología -y todo lo que ella comporta- no puede ser asumida como un medio, dado que es posible encontrar su justificante en sí misma. "El desarrollo de la tecnología desde el siglo XIX ha llegado a ser tan grande que casi nada [por no decir nada] permanece ajeno a ella (...) Lo que es un medio en un contexto, se convierte en un fin en otro" (Hood, 2004, p. 485).

La tecnología existe en razón de sí misma en la medida, en que se la considere como aquella dimensión humana que guarda en sí todo el impulso transformador y productor del ser humano; recordemos que este no cuenta con otra fuerza propia con tal poder de modificación del medio y que, en tal sentido, resulta ser una suerte de mediadora entre el hombre y la naturaleza, entre el mundo interno del primero y el exterior, con miras a proyectar sus fines en este último.

" $<$ Technology $>$ is our primary interface with the other; it is that which lies between the individual and much of experience”. $[<$ Tecnología $>$ es nuestra interface primaria con el otro; esto es, lo que se ubica entre el individuo y el resto de la experiencia] (Murray, 2007, p. 1). 
En ella se encuentran la primera y la última manifestación externa del hombre, el primer impulso volitivo de transformación y el último diseño de avanzada, de modo que hay un continuo en aquélla, y este es el de ser la apertura real a las posibilidades de lo humanamente viable.

Por lo tanto, "frente a esta complejidad y dominio ya no tiene sentido mantener que la técnica [tecnología] es un simple instrumento neutral, que le sirve al hombre para bien o para mal" (Hood, 2004, p. 490). Es, por ende, necesario trascender la visión utilitarista, tradicionalista y estrecha de lo que la tecnología es y forjar una noción lo suficientemente amplia, de modo que nos dé cuenta de manera integral sobre el ser de la tecnología y cuán inmediata, cercana, esta se encuentra de lo humano.

Ante lo anterior, apelamos a Heidegger, como puntal argumentativo, pues coincidimos con su idea sobre la tecnología y a partir de la misma, será seguramente, posible el ver con mayor claridad la noción que sobre la tecnología requerimos para su adecuada comprensión.

Su tesis fundamental al respecto es:

la idea de que la técnica es una parte de la estructura existencial del ser humano. El hombre no permanece en una relación externa con la técnica - es decir, esta no es algo aparte de su ser. La técnica se basa en el hombre--. Por lo tanto, la relación entre el hombre y la técnica puede ser entendida, y la estructura de la técnica fijada, solamente en relación con el ser del hombre (Hood, 2004, p. 490). 
Es decir, el ser humano no es él y la tecnología. No son dos concepciones ajenas, no son entidades que tengan participación óntica en un ámbito de exclusión, sino que la sola mención de lo humano, traería en sí de manera inherente la noción de la técnica, aunque esta, derive de aquel.

Sería, en términos kantianos, y para verlo desde un punto de vista un tanto más abstracto, la emisión de un juicio analítico. Lo tecnológico está de suyo en lo humano. El predicado está de manera implícita al sustantivo.

Pero, ¿cuáles son los espectros de lo tecnológico?, pues "ser es siempre el ser de un ente. El todo del ente, según sus diferentes sectores, puede convertirse en ámbito del descubrimiento y la delimitación de determinadas regiones esenciales" (Heidegger, 2002, p. 32).

Es decir, baste con "desdoblar" desde el ejercicio analítico, todo aquello que se encuentra aparentemente contraído, y con ello, encontrar las reales extensiones, los límites fronterizos que limitan la extensión fundamental de aquello que nominamos Tecnología. Contracción ubicada en el interior del ser humano, pendiente por transitar desde el ámbito de lo oculto, al ámbito de la determinación del ser desde lo ente externo.

Desde lo anteriormente discutido, entonces, surgen las siguientes cuestiones: ¿cuáles son en el caso de la tecnología las regiones esenciales, que nos permitirán dilucidar algo sobre la misma?, ¿cuáles son esas características propias de lo tecnológico?

Para tal efecto, Heidegger afirma son cinco categorías ónticas: "útiles herramientas, instrumentos, aparatos, máquinas-, productos -bienes consumibles y no consumibles-, naturaleza -energías y materiales- 
teorías -el papel de la ciencia-e intersubjetividad-la organización social del trabajo-" (Hood, 2004, p. 494).

A nuestro juicio, faltaría una categoría más, que nominamos exoproyección, es decir, la manifestación que transforma y produce, y que, con ello, plantea una nueva posibilidad sea ya concreta o abstracta al orden, al mundo simbólico de lo humano.

Tomando en cuenta este último punto añadido, consideraríamos, son los aspectos fundamentales -en referencia a la tecnología- a través de los cuales es que el ser de la existencia humana se desenvuelve a través de la creación tecnológica, o dicho en otra palabras, en toda creación de tal naturaleza es posible identificar como elementos en común, lo que los hace esenciales, los anteriormente señalados y es a través de ellos y solo ellos, que la existencia humana proyecta su ser fundamental en el hacer, en el moldear, y en instancia prima, en el idear.

A medida que la interacción entre el hombre y la técnica crece en complejidad, actualizando con ello la dimensión ontológica del hombre, nuestra experiencia cotidiana recibe un carácter cada vez más definido. La tecnología, entonces, es una estructura dinámica que desborda, complica e invade nuestra experiencia cotidiana (Hood, 2004, p. 494).

Es efectivamente, que el ser fundamental en el hacer del hombre se complejiza a través de su devenir, lo que provoca que la interacción entre él y lo que hace, y con lo que dice sobre el cómo hacer, se diversifique en posibilidades cada vez más complejas, lo que genera que al contrario de lo que señala Hood, la experiencia cotidiana en vez de recibir un carácter 
más definido, sea sumamente compleja debido, precisamente, a la indefinición ante tanta posibilidad.

Cabe señalar que las palabras de Hood pueden tomar el rumbo anteriormente señalado, o bien tal vez se quiera referir a tal definición en la medida en que con la actualización la dimensión óntica del hombre, aquello que solo estaba en potencia, lo estaba, por tanto, indefinido, y toda vez que ha pasado al acto, queda ahí, no oculto, definido en cierto aspecto.

Por otra parte, la tecnología en tanto que provocación humana, es tan forzosamente dinámica, que, en efecto, invade nuestra experiencia cotidiana, pero habría que cuestionar en qué sentido es que complica la misma, pues bajo cierta óptica, acaece lo contrario.

Además, lo que es definido, ¿por qué habría de complicar? Lo anterior se menciona a partir de que Hood, que al punto no se sabe si habla por él, o pretende a través de él hacer hablar a Heidegger, señala líneas arriba que nuestra experiencia cotidiana recibe un carácter cada vez más definido toda vez que se actualiza la dimensión óntica del hombre, empero, explica enseguida, que la tecnología complica e invade nuestra experiencia cotidiana, entonces la pregunta es: ¿se complica o no nuestra experiencia cotidiana?

Lo que es definido, no tiene porqué complicar, pues precisamente lo que complica es aquello que se muestra turbio, enmarañado, sin una acotación o simplicidad en sí. Lo definido lleva en sí la seguridad, la certeza de lo que es, por lo que no deja lugar a la complicación, o al menos, no debería ocurrir así. 
Considerando tales categorías como fundamentales ónticos de la tecnología, podemos observar que el orden de todo lo humano se encuentra inserto en ellos, pues en efecto, el humano utiliza implementos que son útiles y a través de los cuales configura su manera de ser y de hacer; de ser y hacer aquello que habrá de consumir a partir de la extracción o generación y manipulación de la energía y la naturaleza.

Haciendo esto último a partir del saber que sobre ella posee desde las teorías científicas y tecnológicas y lo todo lo señalado anteriormente, solo es posible a partir de los sujetos que hacen, que trabajan en un marco de interacciones dadas en una estructura social establecida. Pero detengámonos a fin de analizar detenidamente las categorías mencionadas líneas arriba.

El ámbito fundamental a través del cual se desarrolla la existencia del humano, es el de lo cotidiano. Su existencia se sucede en una constante confrontación ante sí, ante el medio y ante los otros, en la que el continuo es el día a día, caracterizado por "una gama indeterminada de interacciones con las cosas en un contexto, por un repertorio indefinidamente extenso de cosas que (sic) hacer y padecer en algún lugar, en algún momento y con algo" (Hood, 2004, p. 496); pues, dentro de este marco, que se realizan actividades ordinarias que eventualmente permiten el paso a las extraordinarias.

Pero para retomar la idea de lo ordinario, es en este primer orden en el cual la categoría primera señalada por Heidegger en la cual se sitúan los artefactos útiles, "artefactos creados y empleados para la ejecución de propósitos humanos" (Hood, 2004, p. 496). 
Pero por más simple que parezca dicho ámbito de lo cotidiano y sus utensilios, estos son desde su concepción una manifiesta forma del ser, del ser del hombre y de su noción de mundo. Y es en el orden anteriormente mencionado en el que "el ser humano se topa con los útiles y emplea útiles en casi todas sus interacciones con el entorno, y tales interacciones siempre están mediadas por útiles" (Hood, 2004, p. 496).

La relación consigo mismo, con los otros y con el medio (Naturaleza) está mediada a través de aquello que Heidegger llama "equipamiento" (Hood 2004, p.496), que permite la continuidad humana en el orden de la pragmática.

En el andar humano en el mundo, el encuentro con el ser o alguna de sus manifestaciones se extiende a través de los utensilios, los procesos y las ideas, así, "nuestro encuentro con un útil se expresa inicialmente bajo la forma de una intención de llevar a cabo alguna interacción definida con nuestro entorno [o con los otros], tal como está implícito en el uso de ese artefacto" (Hood, 2004, p. 498).

Toda vez que un "útil”, es preconcebido se lo es para cumplir una finalidad, y es en esta medida en que aquél, es decir, el "útil”, está inexorablemente condicionado a modificar el medio en el cual se habrá de encontrar, sea ya de manera "trivial" o de manera relevante; el impacto en el medio provocado por una mesa o un perno metálico, no será el mismo que el provocado por la detonación de una bomba atómica o la construcción de una presa.

Es en el sentido anterior, que dicha modificación del medio es generadora de contextos, de nuevos contextos, por llamarlos de alguna manera 
contextos tecnológicos y, porqué no, circunstancias tecnológicas, y en tal tenor parafrasear a Ortega y Gasset y decir yo soy yo y mis tecnologías.

Es pues el "útil" un espectro de posibilidades, eso sí, siempre estas referidas a las posibilidades de lo humano, de aquel que puede decir de sí que es un ser-en-el-mundo, uno que puede decirse, un siendo ahí, que puede autonominarse como un-ser-para-la-muerte, o mejor aún, como unser-para-la-tecnología. Un fin nunca lo es, si no es este consumado, materializado.

La tecnología "hace posible objetivar y descargar los fines humanos, y expresar la intencionalidad de la experiencia cotidiana cristalizando el hecho de que el ser humano realiza una apuesta al mantenerse a sí mismo, y a su mundo desde la experiencia cotidiana, mediante las cosas" (Hood, 2004, p. 498).

Desde lo ente, la experiencia fundamental humana es con las cosas, natura, consigo y con los otros. La distinción entre dichas experiencias radica en que las cosas (no naturales) derivan de las pulsiones, valores, intereses y necesidades que en determinado momento son primordiales en la experiencia humana. Si nos adentramos en lo más profundo de la experiencia cotidiana podremos identificar una serie de cuasi innumerables objetos que no proceden de physis pero sí, de poiesis. Así:

cualquier ocasionar para aquello que siempre pasa y avanza desde lo no-presente a estar-presente es $\pi$ oin $\sigma 1 \varsigma^{7}$, es un producir (Her.vorbringen). Todo reside en que pensemos el producir en toda su amplitud y, al mismo tiempo, en el sentido de los griegos. Un 


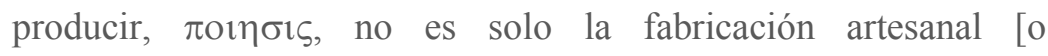
tecnológica], no es el acto poético-artístico de llevar-a-modelos-y-aimágenes. También la $\varphi v \sigma l \varsigma^{8}$, el salir-desde-sí (das von-sich-her-

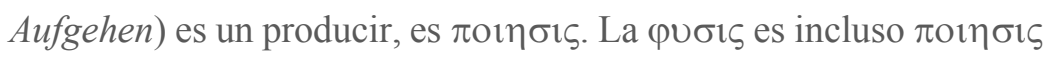
en el más alto sentido. Pues el $\varphi v \sigma \varepsilon ı$ que está presente tiene en sí mismo $\left(\varepsilon v \varepsilon \alpha v \tau \varpi^{9}\right)$ la apertura del producir (por ejemplo, el abrirse de la flor en el florecer). Por el contrario, lo producido artesanal, [tecnológicamente], o artísticamente no tiene la apertura del producir en sí mismo, sino en otro (...), en el artesano, [el tecnólogo] o el artista (Heidegger, 1990, p. 134).

Y es en este ámbito de cosas, en lo producido por el otro, en el cual se contextualiza toda vivencia humana, ninguna experiencia humana está exenta del contacto por lo hecho por otro ser humano. Aquí cabe señalar que ante la noción de la "técnica moderna", Heidegger desentiende el concepto de $\pi$ oin $\sigma \iota \varsigma$ de esta, pues "el desvelamiento imperante en la técnica moderna es un provocar (Herausfordern)" (Heidegger, 1990, p. 137) Mas tal provocar no es distante, metafísico; inherentemente conlleva un transformar, un producir. La exigencia por la energía tiene, dínamos, ductos y canales bien definidos, desde los cuales, se suministra el enérgico necesario que permite el desdoblamiento de la vida, pero desde los cuales, el mismo ser se manifiesta.

Dicho lo anterior, y retomando el hilo de la discusión, y ante nuestra actual circunstancia, ante nuestra más prístina vivencia, cómo no podríamos cuestionar si no es que, en un atrevimiento de nuestra parte, preguntar si es acaso que, ¿no es el humano un ser-para-la-tecnología? Y cómo no cuestionar lo anterior si al momento en que se redactan estas líneas, no solamente son los dedos lo que lo hace posible, sino una serie de elementos 
tan disímiles entre sí que resulta complejo no encontrar en la convergencia de todos ellos una finalidad. Pues, ¿qué tendría qué ver el coltán ${ }^{10}$ congoleño con la corriente eléctrica o las fallas estructurales por corte? Aparentemente nada, empero, tiene que ver todo en la conformación de un contexto que ante nuestra actualidad trasciende lo particular y se torna generalizado.

Finalmente, y en términos allende los particulares, nos encontramos cada uno de nosotros insertos en espacios diseñados, construcciones de acero o concreto, o de ambas, que han sido preconcebidas y sujetas a diversos análisis a fin de que sean estas seguras.

$\mathrm{Y}$ es dentro de tales espacios, en los que utilizamos los equipos de cómputo, que conectamos a la red eléctrica que se despliega por las entrañas de la construcción, como si fuera una suerte de sistema circulatorio que lleva la energía a donde esta es requerida. Dicha corriente eléctrica es la que proporciona el "alimento" a nuestros equipos electrónicos, que en su interior muchos de ellos, llevan capacitores elaborados a base de coltán.

Convergen pues, la multiplicidad de posibilidades en un concreto a partir del cual, se generan, se crean contextos vitales, experiencias de vida y con ello, de entender y enfrentar el mundo. ¿No es pues la tecnología un bloque constitutivo fundamental del muro en la construcción del ser?

La conformación del ser no es un asunto de carácter estrictamente personal o particular, sino que resulta ser el reducto convergente de una diversidad de circunstancias a través de las cuales el que se dice "yo", se experimenta 
a sí mismo. En esta experiencia están presentes inevitablemente los "útiles", los procesos y las ideas; preconcebidos, diseñados y finalmente materializados para ser recibidos y utilizados en un sentido u otro.

Mediante el proceso de crear útiles para tratar con los entes, el hombre se experimenta a sí mismo como el creador de su actividad permanente, pero también como aquel que es receptor; se descubre, pues, a sí mismo como condicionado y como condicionado mientras se mueve entre los entes de su experiencia cotidiana (Hood, 2004, p. $501)$.

Es por lo anterior que el sujeto inscrito dentro de este perímetro de cotidianeidad pierde de vista el contorno de lo extraordinario, y de la posibilidad de observarse, de rozar el ser a través de la no sola utilización, sino a través de la contemplación de los objetos en derredor suyo, con miras a la comprensión de estos, que, en última instancia, le pueden acercar a la comprensión de su ser-siendo, y con ello, de cierto fragmento de la realidad.

Mas lo anterior ha de ir más allá, pues si bien es cierto que a través del uso de los "útiles" el humano penetra en su propia esfera, también es cierto que a través de estos "el hombre penetra en la naturaleza, descubre un ámbito no humano, y lo modifica de manera que pueda constituirse como un mundo humano" (Hood, 2004, p. 502). Dichos útiles no son otra cosa sino tecnologías, mediante las cuales el ser humano, además, escudriña, indaga lo que aquélla, es decir la naturaleza, no muestra por sí misma o en todo caso, resulta inalcanzable a las facultades físicas propias del ser humano. 
Téngase en cuenta, y a modo de ejemplo por antonomasia, las posibilidades alcanzadas a través del telescopio o del microscopio. Pero lo anterior no basta, existe también la exigencia de la manipulación, ¿y qué otra cosa no es lo que manipula el ser humano, otrosí de la materia, sino la energía? Es decir, la posibilidad del movimiento, del flujo.

"La naturaleza no está solamente alrededor del hombre material, sino también frente a él como energía. La naturaleza ofrece una dirección para la ejecución de tareas; sugiere al hombre cómo podría hacer mejor uso de su entorno" (Hood, 2004, p. 502). Mas el entorno natural es cambiante y con ello las posibilidades diversas ante las cuales el humano, y específicamente el que transforma, el que produce, debe elegir. El qué lo lleva a elegir o el por qué, no es objeto de discusión de la presente investigación, de modo que únicamente asumamos que lo hace.

¿En qué momento el hombre tendió un puente entre la naturaleza y la tecnología? O tal vez la pregunta tendría que plantearse en los términos siguientes: ¿en qué momento el ser humano se incorpora a la naturaleza y encuentra en esta algo que no es de suyo? O bien, ¿en qué momento se escinde este de aquélla y la contempla como algo a lo que si bien, perteneció, ahora le es infamiliar?

Con certeza plena no lo podemos saber, pero lo que sí podemos afirmar en este ahora, es que hay un afuera saturado de, ya no solo naturaleza, sino de objetos y hechos de los cuales la naturaleza por sí sola, no puede o podría dar cuenta. "Desde el punto de vista físico o el ingeniero, la naturaleza es un conjunto calculable de fuerzas y nada más” (Hood, 2004, p. 504). 
Fuerzas que son solamente calculables y nominables por el hombre, y un tipo particular de hombre, el docto, el instruido, el que ha sido capacitado, entrenado para recibir y desarrollar cierto tipo de saber que guarda en su interior el de la posibilidad de transformar y producir.

Es en este ámbito de lo humano en el cual está presente la intersubjetividad. En otras palabras, la capacidad de comunicación del conocimiento dentro de una comunidad científico-tecnológica o de otra naturaleza. Y es que resulta necesario hablar de otras comunidades y no solamente de las científico-tecnológicas, debido a que según Heidegger es posible distinguir dos tipos de personas, a saber:

aquéllas que saben cómo usar los artefactos en sus respectivas totalidades contextuales. Una condición necesaria para que algo se convierta en útil es que alguien sepa para qué está hecho en una totalidad contextual dada; $y$, análogamente, una condición necesaria para que algo sea un producto es que alguien vaya a usarlo. Naturalmente, en muchas situaciones un individuo será simultáneamente usuario y consumidor (Hood, 2004, p. 506)

Lo anterior nos lo señala Hood a partir de cierta noción heideggeriana, que eventualmente nos parece podemos encontrar en un autor posterior como lo es Langdon Winner, quien en su texto "La ballena y el reactor" (1987) nos dice no sin decepción:

que heredamos de tiempos lejanos -a partir de un punto de vista convencional- [que] la relación humana con los objetos técnicos es demasiado obvia para merecer una reflexión seria. (...) La gama de posibles intereses [en que es dividida] acerca de la tecnología en dos 
categorías básicas: hacer y utilizar. En la primera la atención se centra en "cómo funcionan las cosas" y en "hacer que las cosas funcionen". Tenemos la tendencia de pensar que esta es una atracción para ciertas personas en determinadas ocupaciones, pero para nadie más. “Cómo funcionan las cosas?” es el terreno de los inventores, los técnicos, los ingenieros, los mecánicos de reparaciones, etc., quienes preparan instrumentos artificiales para la actividad humana y los mantienen en buen funcionamiento. Se piensa que aquellos que no están directamente involucrados con ninguna de las esferas del "hacer" tienen poco interés o necesidad de conocer los materiales, los principios o los procedimientos que incluyen dichas esferas (Winner, 1997, p. 21).

Es en simples y llanas palabras, quién hace y quién usa. El que proyecta, el que hace, transforma y en resultado obtiene cierta modificación de su entorno, no solamente físico, sino circunstancial.

Es clara la distinción que existe entre quien hace y entre quien "hace uso", entre quien de la poiesis se vuelca con fines de posibilidad de extensión del ser, en tanto que nueva manifestación del mismo, en un principio eidético, y finalmente materializado, que se traduce en la posibilidad y en la extensión tangible del predicado del ser.

Se piensa con antelación la posibilidad, a partir de un referente simbólico, y se avizoran a priori los diversos escenarios de dicha posibilidad. Es decir, de lo uno en ahí, y de un predecesor que lo hubiera concebido y llevado de la idea al carácter de la tangibilidad. Es con esto último que lo asequible, lo que tiene la tangibilidad como potencia, se nos muestra en una tarea de 
transformación, de producción y de la experiencia de lo sensible, en un marco de signos, y en la experiencia de lo intelectivo, en un universo simbólico, y con esto es que "la adquisición del signo en el desenvolvimiento inmanente del espíritu constituye en verdad un primer y necesario paso para la conquista del conocimiento esencial objetivo" (Cassirer, 1971, p. 31).

Así, con la adquisición de dicho conocimiento, es que la realidad humana morfa, "crece" en términos de sus posibilidades y con ello, las extensiones del ser en abstracto, y del ser en lo concreto.

Así, los objetos, las ideas y los procesos están ahí, mostrando su ser, sea ya en potencia, sea ya en acto, y con ello, el ser de otro y de los otros, y en un mutismo, pasando por alto la indiferencia a la que estos últimos hubieron hecho caso omiso.

"Trabajar con otros hace asequible el entorno ayudando a establecer en él la matriz de totalidades contextuales" (Hood, 2004, p. 507). Conocemos en prima instancia gracias a los demás, en la medida en que somos "sumergidos" en las aguas del mar simbólico, en la medida en que aprendemos a nadar en ellas y al salir de estas, ir en búsqueda de nuevas aguas que amplíen nuestro bagaje cognitivo, es que identificamos y ampliamos los contextos humanos.

Podemos concretar el punto último de las categorías ónticas que Heidegger finca como primordiales sobre el pensar la tecnología con lo señalado en la cita anterior, y en general decir sobre las cinco, más la exo-proyección, que, como tales: 
son elementos de la estructura existencial del ser humano; y, en tanto que concretados y percibidos como realidades objetivas, se encuentran también fundados en su ser. Es en el contexto de tales condiciones trascendentales, más que como objetos de una teoría aristotélica, como pueden establecerse los límites de la técnica (Hood, 2004, p. 508).

En otras palabras, lo tecnológico no es solo un fútil medio, allende eso, resulta ser lo más próximo a lo humano como fin en tanto que está en él mismo, como especie, y no en la physis. Mas para que se desentienda del ámbito del "no estar", del no ser, es necesario que se dé la "liberación ontológica [que] es previa a la óntica porque un útil debe existir antes de que pueda ser el útil que es" (Hood, 2004, p. 508). Dicha liberación ontológica se gesta, en un primer momento, en el interior de la imaginación, para posteriormente arribar al ámbito de lo óntico toda vez que cobra su carácter de estar ahí, y con ello, de útil, sea ya en objeto, en proceso o idea.

Así, "liberando útiles el hombre libera igualmente, productos, naturaleza, teoría y organizaciones de personas” (Hood, 2004, p. 509). Es, así pues, en este liberar que cada ente entra en interacción con los otros entes de las diversas esferas señaladas, conformando el total, el conjunto omnipresente que llamamos realidad, en la que todo lo liberado tiene su ser ahi y en la que el ser humano se proyecta y multiplica el ser, de lo que:

se sigue que el significado ontológico de la técnica no es el que haga posible la satisfacción de las necesidades humanas a través del conjunto de medios más eficiente, o que algo instrumental en el 
sentido más amplio de la palabra -ambas verdades son triviales y expresan solamente el lado inauténtico del hombre-, sino el que refleja el cuidado del hombre, el hacerse cargo de, que el hombre tiene por el Ser de todos los entes; representa una forma mediante la cual el hombre expresa su receptividad, y no exclusivamente su domino, hacia las cosas en la totalidad del mundo (Hood, 2004, p. 511).

Una vez leído lo anterior, volvemos a nuestra pregunta inicial, fundamental y es esta el preguntar por la tecnología, a saber, ¿qué es la tecnología?, ¿ha sido posible a través de nuestra perorata dilucidar al menos un esbozo de lo que la tecnología es?

No pretendo a través del cuestionamiento anterior otorgar una respuesta definitiva, ni mucho menos, sino que se extiende el cuestionamiento con el fin de no extraviarnos sobre lo que por discusión se lleva, y la discusión es sobre ¿qué es la tecnología?

Y al hacernos esta pregunta cuestionamos el sentido ontológico de ella, es decir, por el ser del ente llamado tecnología, y extendemos la pregunta sobre si el acaso es posible la antinomia, es decir, si cabe la posibilidad del ser humano que no deje algo de sí, que no se plasme a través de ése, su hacer, su transformar y producir.

¿Puede permanecer inerte, inmóvil?, ¿estéril, en tanto que especie, y en tanto que no pretenda transformar y producir?, ¿puede ser neutral ante su hacer, neutral ante su proceder? 
Ahora el hombre, como Heidegger lo contempla, está implicado en las interacciones con los útiles de una manera que refleja su propio cuidado por ir más allá de cualquier naturaleza específica fijada que pudiera tener y de la situación particular en la que ahora pudiera encontrarse. Las interacciones del hombre con los artefactos pueden verse ahora como reflejo de su capacidad para estar abierto a lo que yace más allá de sí mismo y para trascender su propia naturaleza (Hood, 2004, p. 512).

La respuesta al planteamiento último, nos la ofrece Heidegger en palabras de Hood, y esta es que no, no puede ser neutral el ser humano en su transformar, en su producir, en su tecnología. "The 'technology' we are discussing is not, and cannot be, morally 'neutral'”. [La 'tecnología' que estamos discutiendo no es, y no puede ser, moralmente 'neutral'] (Black, 1976, p.187). La misma interacción con los útiles, refleja su propio cuidado.

Ahora bien, se señala que "Las interacciones del hombre con los artefactos pueden verse ahora como reflejo de su capacidad para estar abierto lo que yace más allá de sí mismo y para trascender su propia naturaleza".

Pero, ¿si es el caso que la naturaleza propia del humano es la de un ser tecnológico, en qué medida es que dicha interacción con los artefactos permitiríale trascender su "naturaleza"; no más bien estaría en el camino de su propia teleología y con ello, cumpliendo a cabalidad su ser en tanto que "ser para"? 
"Los cinco sentidos, la movilidad física, la fuerza y la capacidad de acción del hombre han aumentado $\mathrm{y}$, desde ciertos puntos de vista, han mejorado gracias a ella [la tecnología]" (Winner, 1979, p. 179). Aquí la pregunta que consideramos necesaria es, ¿acaso podría haber sido de otro modo?

Es decir, ¿pudo el ser humano no haber desarrollado todo esto que ha permitido inyectar mayor celeridad a nuestro desplazar, mayor fuerza a la propia de nuestra vitalidad y en pocas palabras llevar nuestras acciones allende las fronteras a las que nos encontramos circunscritas en términos de naturaleza biológica?

Desde este punto de vista la técnica es importante, por permitirnos hacer cosas que antes nos hubieran sido imposibles. Quizá es más exacto decir que permite a los individuos ser algo, una forma de ser que antes era desconocida sobre la tierra. Una consecuencia de la extensión es que, de un modo nunca visto con anterioridad, no es precisa la presencia física de una persona para que se produzca una acción. Todas las variedades de control, expresión pensamiento, movimiento y producción de acontecimientos pueden realizarse por medios lejanos que cuentan con líneas de gran longitud. Los individuos pueden ahorrarse «estar presentes», gracias a una serie de inventos más o menos complejos. La lejanía es, por consiguiente, una consecuencia de la extensión (Winner, 1979, p. 179).

Al parecer es de toda necesidad, entendida esta en términos aristotélicos, como aquello que no puede ser de otra manera, que inexorablemente estamos como especie proyectados hacia el devenir tecnológico, asociando nuevas posibilidades de acción, a nuevas formas tecnológicas: 
incluso pueden clasificarse las técnicas según cuál sea la capacidad humana en la que influyen y de qué modo lo hacen. Los medios de transporte aumentan nuestra capacidad de movimiento en el espacio geográfico. Los medios de comunicación extienden nuestras posibilidades de ver, ori, hablar y expresarnos a grandes distancias (Winner, 1979, p. 179).

Pensar, es un camino, señaló Martin Heidegger. Un camino que una vez iniciado, no finaliza sino y con aquello que él mismo apuntó de una manera tan contundente que no cabe la dubitación: el hombre, es un ser-para-lamuerte. Y en la muerte, con tal morir, finalizan las posibilidades todas del hombre, pero se abre una más, es esta, la de la trascendencia. Pero mientras vive, hace; transforma y produce, esto, en tanto que especie.

El trabajo que se ha presentado ha sido el reflejo de un pensar iniciado, de un recorrido comenzado, de un camino que se ha empezado a transitar y que, a través del mismo, se ha procurado clarificar el quid de aquello que, como especie humana, hemos hecho a lo largo de ya tantas centurias, a saber, el cultivo tecnológico, el transformar y producir. ¿Qué es?, o para decirlo de otra manera, ¿qué es la Tecnología?

Es la pregunta prístina que se ha planteado a lo largo del presente estudio, y que ha sido respondida de manera parcial, y por ende no saldada, desde una perspectiva filosófica, procurando la mayor claridad posible en la misma, aunque si bien, como todas las respuestas filosóficas, no guarda en sus palabras la imposibilidad de verse superada. Sí ofrece, a nuestro juicio, un punto de continuidad radical sobre la respuesta dada a la pregunta por la tecnología, hasta hoy día planteada. 
El responder filosóficamente una cuestión, no es únicamente despliegue de erudito bagaje, sino el producto, el resultado de una madurez, que, como la vid desde que es sembrada, y hasta que ofrece la primera cosecha viable para elaborar el buen vino, requiere de tiempo. Tiempo en el que se analiza y se sintetiza lo analizado para volver esto un proceso iterativo; dialéctica del análisis y la síntesis, añadiendo a modo de complemento, el conocimiento pertinente y disponible al momento de realizar la investigación.

Hacer un esfuerzo genuino por ofrecer una respuesta integral y coherente, mas no acabada, a la no sencilla pregunta de ¿qué es la tecnología? representa en la actualidad un imperativo, un hacer que no se puede postergar más, debido a las repercusiones que el ejercicio tecnológico ha tenido, tiene, y habrá de tener, sobre la especie y el lugar que la especie ocupa en la existencia, es decir, el mundo.

Es la dimensión del ser en la que se proyecta su ansia de plenitud existencial; la que concreta sus aspiraciones máximas y en la que su interior exterioriza su álgida experiencia vital, que es, no la creación ex nihilo, pero sí, la creación de su propio mundo, del que se siente orgulloso, del que se vanagloria tras colocar la última piedra, la dimensión de su autoconstrucción y de su proyección óntica, a partir del transformar y producir. Provocando con ello, un cambio, una transformación del antropocosmos, que redirecciona a este y es así, que, por ejemplo, "una tecnología puede cambiar la propia forma en la que la comunicación lingüística puede tener lugar, y así, producir un cambio en el propio mundo de la vida" (Ihde, 2009, p. 156). ${ }^{11}$ 
¿Qué es Tecnología? Una aproximación desde la Filosofía...

Es, por tanto, un rasgo fundamental del ser humano la transformación, esencial en tanto que humano y en vida, como especie, su desde-el-serpara-transformar-y-producir. 
Black, M. (1976). Are there any Philosophically Interesting Questions in Referencias Technology? PSA: Proceedings of the Biennial Meeting of the Philosophy of Science Association. Symposia and Invited Papers, pp. 185-193. The University of Chicago Press. Recuperado de: http://www.jstor.org/stable/192381

Bunge, M. (2004). Acción. En Mitcham, Carl \& Mackey Robert (Eds.), Filosofía y tecnología, (pp. 63-92). Madrid: Ediciones Encuentro.

Cassirer, E. (1971). Filosofía de las formas simbólicas [Volumen 1]. México, D.F.: Fondo de Cultura Económica.

Chiappe, A. (2009). La posibilidad de un Puente sobre problemas actuales de la filosofía de la tecnología. Utopía y praxis latinoamericana, 46, pp. 51-69.

González, J. (coord.). (2008). Perspectivas de bioética. México, D.F.: UNAM, Comisión Nacional de los Derechos Humanos y Fondo de Cultura Económica.

Heidegger, M. (1990). La pregunta por la técnica. En Anales del Seminario de Metafísica, 24, pp. 129-162. Madrid: Ed. Universidad Complutense.

Heidegger, M. (1994). Conferencias y artículos. La cosa. Barcelona: Ediciones del Serbal.

Heidegger, M. (1997). Introducción a la Metafisica. Barcelona: Editorial Gedisa.

Heidegger, M. (2007). La pregunta por la técnica (y otros textos). Barcelona: Ediciones Folio, S.A.

Hood, W. (2004). El problema de la técnica: el enfoque aristotélico versus el heideggeriano. En Mitcham, Carl \& Mackey Robert (Eds.). Filosofía y tecnología, (pp. 479-512). Madrid: Ediciones Encuentro.

Kapp, E. (2001). Líneas fundamentales de una filosofía de la técnica. En López, C. José A. et al. (Eds.). Filosofía de la tecnología, (pp.125133). Madrid: O.E.I. 
Lee, K. (2009). Homo faber: the Unity of the History and Philosophy of Technology. En Berg Olsen, Jan Kyrre, Selinger, Evan \& Riis, Soren. New Waves in Philosophy of Technology. (pp. 13-39). London: Pagrave Macmillan.

Mitcham, C. \& Mackey, R. (Eds.). (2004). Filosofía y tecnología. Madrid: Ediciones Encuentro.

Murata, J. (2006). From Challenger to Columbia: What lessons can we learn from the report of the Columbia accident investigation board for engineering ethics? Techné: Research in Philosophy and Technology, 10 (1), pp. 35-52.

Murray, C. (2007). The Project of Technology. Tesis de Doctorado en Estudios Humanísticos con Especialidad en Ciencia y Cultura, no publicada. Escuela de Humanidades y Ciencias Sociales, Tecnológico de Monterrey, Campus Ciudad de México.

Ropohl, G. (1997). Knowledge Types in Technology. International Journal of Technology and Design Education, 7, pp. 65-72. Recuperado de: http://course.zjnu.cn/kcjx/uploadfile/20081127194457977.pdf

Winner, L. (1979). Tecnología autónoma. Barcelona: Editorial Gustavo Gili, S.A.

Winner, L. (1987). La ballena y el reactor. Barcelona: Editorial Gedisa S.A. 
1. Por esencial nos remitimos aquí a la noción aristotélica de esencia, es decir, aquello que hace que la cosa sea lo que es y no otra cosa.

2. La tecnología en la medida en que posee una dimensión del hacer del hombre, es susceptible de valoraciones de carácter ético. A pesar de que el objetivo de la presente investigación no transita por tales caminos, un estudio de caso relevante al respecto, puede ayudar a clarificar el por dónde se encamina la discusión en relación a la ética y tecnología. Sugerimos al lector revisar: Murata, Junichi. (2006). From Challenger to Columbia: What lessons can we learn from the report of the Columbia accident investigation board for engineering ethics? Techné: Research in Philosophy and Technology. 10, (1), pp. 35-52. En donde las cuestiones de seguridad dejan claro el artículo, pasaron a segundo término, ante la premura de los administradores de la NASA por el cumplimiento de las misiones. Por otra parte, y ante los desarrollos más recientes en materia de genética y en general, de la manipulación de la vida, en el amplio espectro que esta implica, la bioética ha venido a posicionarse como una disciplina joven y en amplio desarrollo por las implicaciones inherentes a dicha manipulación. Por lo anterior se considera que el conocimiento, aunque sea de los problemas generales que se plantean en la Bioética, deben ser objeto de estudio del filósofo de la tecnología. Un excelente marco introductorio se lo puede encontrar en: González Valenzuela, Juliana. (coord.) (2008). Perspectivas de bioética. México, D.F.: UNAM, Comisión Nacional de los Derechos Humanos y Fondo de Cultura Económica.

3. Léase tekne.

4. Léase logos.

5. Entiéndase lo artesanal como una extensión de lo tecnológico en la medida en que son el resultado de un hacer del hombre, y no una eyección desde sí en la naturaleza.

6. Léase en alloo.

7. Léase poiesis.

8. Léase physis.

9. Léase en eautoo. 
10. El coltán es un mineral óxido, cuyo nombre resulta de la abreviatura de columbita y tantalita. Es utilizado para la construcción de capacitadores, que son componentes fundamentales de los teléfonos móviles y otros artificios electrónicos. Se estima que el $80 \%$ de las reservas mundiales de coltán se encuentran en República Democrática del Congo. Recuperado de: http://news.bbc.co.uk/2/hi/africa/1468772.stm

11. Para profundizar un poco más en el planteamiento fenomenológico de la tecnología, revísese: Ihde, Don. (2005). A Phenomenology of Technics. En Scharff, Robert C. \& Dusek, Val (Eds.), Philosophy of Technology. The Technological Condition. An Anthology. (pp. 507-529). Oxford: Blackwell Publishing Ltd.

Recibido: 23-octubre-2015

Aceptado: 20-febrero-2016

Todos los derechos reservados. Universidad de Costa Rica. Esta revista se encuentra licenciada con Creative Commons ReconocimientoNoComercial-SinObraDerivada 3.0 Costa Rica. (CC BY-NC-SA 3.0 CR)

Correo electrónico: humanidades@ucr.ac.cr Sitio web: http://revistas.ucr.ac.cr/index.php/humanidades 